\title{
Creating a functional basis for the production of food emulsions
}

\author{
Olga Feofilaktova*, Dmitrij Grashchenkov, Dmitrij Karkh, and Mikhail Lukinykh \\ Ural State Economic University, 620144, Yekaterinburg, Russia
}

\begin{abstract}
Occurence dependence of a number of diseases on the breakdown of the population nutrition structure has been established for a long time. Epidemiological and clinical evidence supports the role of functional food ingredients in the pathogenesis of various diseases. The omega-3, $-6,-7$ and -9 fatty acids play a special role among them. Having a number of functional properties, they contribute to the prevention of a number of common diseases, especially cardiovascular diseases. These mono- and polyunsaturated fatty acids are among the most common vegetable oils. This article describes the technology of developing a functional fat base for emulsion food production based on a combination of several types of vegetable oils. The technology uses a specially designed computer program that allows to compose a combination of vegetable oils based on the optimal ratio of omega- 3 and omega- 6 fatty acids, as well as with taking into account the physiological standards of consumption of mono- and polyunsaturated fatty acids.
\end{abstract}

\section{Introduction}

One of the reasons for the development of alimentary diseases, which make up 30 to $50 \%$ of the causes of mortality, is the lack of nutritional structure of the population. Such diseases include diseases of the cardiovascular system, diabetes, osteoporosis, obesity, malignancies [1-3].

Currently at the state level the standards of physiological needs in energy and food substances for different groups of the population of the Russian Federation have been approved, determining the values of physiologically substantiated norms of consumption of food substances, adequate levels of consumption of micronutrients and biologically active substances with established physiological action by modern nutrition science [4].

In order to manage health risks and ensure a balanced sustainable diet of different population groups, it is necessary to include functional food products - special food items intended for the systematic use as part of food diets by all age groups of a healthy population with scientific and proven properties, reducing the risk of alimentary diseases, preventing deficiency or filling nutrient deficiencies in the human body, preserving and improving health through the availability of physiologically functional food ingredients in their composition [5]. In the creation of functional food products, in order to substantiate

\footnotetext{
*Corresponding author: feofiov@usue.ru
} 
their compositions, the scientific basis is the norms of physiological needs in energy and food substances.

Special attention should be paid to physiologically functional dietary ingredients such as mono- and polyunsaturated fatty acids, which are part of most common oils.

\section{Literary review}

\subsection{Functional properties of unsaturated fatty acids of omega groups}

Unsaturated fatty acids refer to functional dietary ingredients due to the fact that they are physiologically active, valuable and healthy ingredients with known physical and chemical characteristics for which the properties useful for preservation and improvement of health have been identified and scientifically substantiated, as well as the daily physiological need has been established.

Based on the requirements of GOST R 52349-2005, according to which a functional ingredient is the one that is capable of providing scientifically substantiated and validated effect on one or more physiological functions, processes of metabolism in the human body with systematic use of functional food products containing them, according to GOST R 54059-2010 all functional food ingredients are classified into classes, groups and subgroups.

According to this classification, omega-3 fatty acids depending on the type of effect exerted on one or more physiological functions, the processes of metabolism in the human body are related to Class A (substrate metabolism effect), Class B (antioxidant effect), and Class E (immune system support effect). Depending on the results of the evaluation of omega-3 efficiency, fatty acids are assigned to group II (carbohydrate metabolism), subgroup 1 (blood glucose levels control), and 2 (maintaining insulin levels in blood); group I (cardiovascular system functions), subgroups 2 (preservation of blood vessel wall tone and their passability), group II (lipid metabolism), subgroups 1 (blood triacylglycerins level control) and 2 (level control of total cholesterol, high and low density lipoproteins in blood) ; as well as group I (immunocorrective action), subgroup 1 (systemic immunomodeling action), grades A, B and E respectfully as stated above. Omega- 6 fatty acids, within class B (antioxidant effect) are characterized by performance results according to group I (cardiovascular function), subgroup 3 ( antithrombotic effect), as well as group II (lipid metabolism), subgroup 1 (blood triacylglycerins level control) and 2 (level control of total cholesterol, high and low density lipoproteins in blood). Monounsaturated fatty acids, including omega-7 and omega-9, depending on the type of effect belong to class $\mathrm{B}$ (antioxidant effect), group II (lipid metabolism), subgroups 1 (blood triacylglycerins level control) and 2 (level control of total cholesterol, lipoproteins of high and low density in blood). [6].

Figure 1 presents the structure of omega groups on the corresponding fatty acids, as well as the types of effects on one or more physiological functions of the organism.

Monounsaturated fatty acids include oleic acid, also known as omega-9-fatty acid and omega-7, or palmitoleic acid (fig. 1). Oleic acid is found in large quantities in olive oil, avocado oil, sesame seed, peanut and argan oils, as well as in canola oil. Oleic acid plays a certain role in metabolism of essential fatty acids, regulation of anti-inflammatory processes [7-9].

Palmitoleic acid is synthesized in the body in the liver and adipose tissue, with insufficient synthesis of this acid leading to the development of a range of pathologies. It is a link between adipose tissue and other organs regulating fat and carbohydrate metabolism. It plays a crucial role in moisturizing the mucous membranes of the internal organs, 
determines the state of the skin, regulating the degree of hydration and affecting elasticity, is able to reduce fat deposition through insulin stimulation and reduction of insulin resistance of tissues vand hinder the development of obesity, contributes to the reduction of inflammatory processes (both internal and external), improves functions of heart, reduces the risk of hypertension and atherosclerosis, promotes normalization of triglycerides levels in the body [10-12].

The sources of omega-7 fatty acids are macadamia nuts. They contain $80 \%$ of monounsaturated fatty acids, where $60 \%$ is oleic acid (omega-9) and $20 \%$ is palmitoleic acid (omega-7).

Palmitoleic acid is also contained in sea buckthorn oil and avocado oil, which is rich in other monounsaturated, polyunsaturated and saturated fats, small amounts of omega-7 fatty acids are contained in olive, wheat germ oil, mustard and other types of oils.

Polyunsaturated (essential) include omega-3 fatty acids and omega-6 groups. Highvalue omega-3 fatty acids are eicosapentaenoic acid (EPC) and docozohexaenoic acid (DHA) due to the fact that the body can accumulate them immediately, without prior adjustment.

In plants, there are predominantly alpha-linolenic acid, which is not as high value because in the body it must first convert to EPC and DHA.

The significance of Omega-3 PUFA is to reduce the risk of cardiovascular disease and atherosclerotic damage, development of coronary heart disease, maintenance of normal brain, eye and nerves development, in normal growth of children, in maintenance of homeostasis of inflammatory reactions, in the formation of highly active immunoregulators, in therapy aimed at prevention of myocardial infarction (fig. 1).
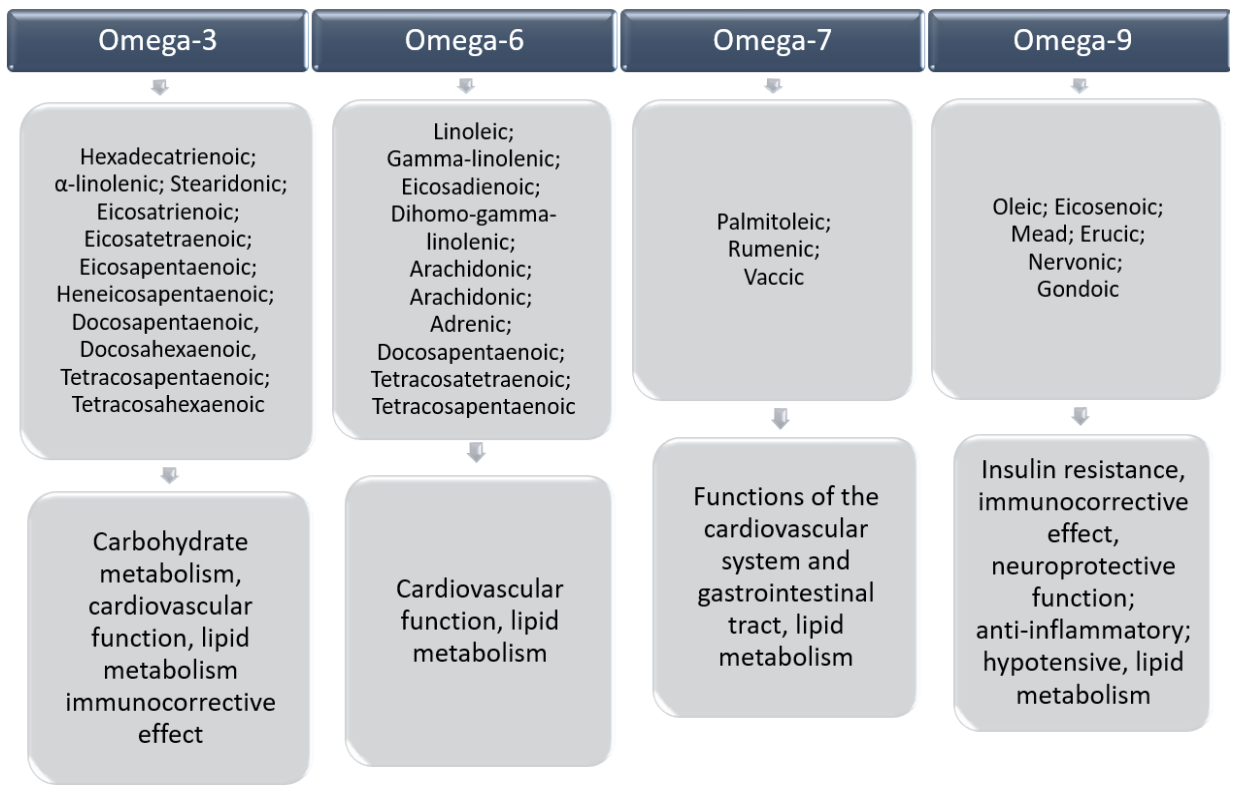

Fig. 1. Structure and functional properties of omega fatty acids.

There is evidence on the effect of Omega- 3 on the preservation and even repair of telomeres in DNA molecules of organism cells and, as a result, an increase in life expectancy $[13,14]$.

Omega-3 acids affect blood coagulability, make up one of the links of fat transformation in the body. By embedding in cell membranes due to the growth of lipid oxidation products concentration, they reduce the electrical instability of the myocardium. To date, a large 
number of studies confirm the effect on ion flows in cell membranes. Influence on sodium, potassium, calcium channels was studied, protective effect on myocardium and prevention of arrhythmias [15-17] was explained.

A clear connection between daily consumption of omega-3 fatty acids and the degree of atherosclerotic lesion of coronary vessels has been established. The more omega- 3 fatty acids are found in body tissues, the fewer manifestations of atherosclerosis. For this reason, the importance of omega-3 PUFA is to reduce the risk of cardiovascular disease and atherosclerotic damage, the development of coronary heart disease.

The data provided give an idea of the Omega-3 family PUFA as an antiatherosclerotic agent and substantiate its use for primary prevention of atherosclerotic diseases [18-19].

Omega-6 family include linoleic acid (LC), gamma-linolenic acid (GLC), dihomogamma-linolenic acid (DGLC), arachidonic acid (AK), etc. (Figure 1). The proven functional properties of omega- 6 fatty acids consist in reducing the risk of myocardial infarction, the level of total serum cholesterol [19]. In addition, the following important effects of their impact on the body can be stated: the protection of cells from external damage and the strengthening of their natural barrier and the fact that essential substances that have anti-inflammatory and anti-allergic effects are synthesized out of them . Arachidonic acid participates in the formation of hormone-like substances regulating the work of genitourinary, cardiovascular, respiratory, immune and nervous systems, gastrointestinal tract [20].

However, the amount of PUFA in the diet needs to be regulated due to the fact that their large doses can increase lipid peroxidation when saturating body tissues. In addition, when omega-6 is oversupplied, they begin to compete with omega-3 for desaturase enzymes responsible for the formation of double bonds and involved in the synthesis of essential acids from other ones. The imbalance in the ratio of incoming PUFA increases the risk of certain diseases - thrombosis, arthritis, atherosclerosis, asthma, immune-inflammatory processes [21].

Therefore, the ratio of omega-3 to omega-6 PUFA in the diet is important.

The relationship between omega- 3 and omega- 6 fatty acids and disease pathogenesis continues to be a topic of extensive study. To a lesser extent, omega-9 and omega-7 fatty acids were seen as potential disease mediators. These fatty acids can work individually, additively, or synergistically as precursors and critical elements within metabolic pathways, actively affecting disease pathogenesis.

\subsection{Physiological norms and food sources of unsaturated fatty acids of omega groups}

Consumption of PUFA by the body depends on lifestyle, place of residence, nature of nutrition.

Based on the results of numerous studies, a group of experts from the American Cardiology Association developed guidelines for the use of EPC/DHA - $300 \mathrm{mg}$ per day for healthy adults, and those with coronary heart disease should take 2-4 g of EPC and DHA in the form of biologically active supplements [22].

The Ministry of Health of Russia recommends $1 \mathrm{~g}$ of alpha-linolenic acid(ALC) /EPC/DHA per day for adequate consumption [5].

The main food sources of PUFA are oily marine fish, fish oil and seafood, crushed flax seeds and linseed oil, mustard and dodder oil, rapeseed oil, soybeans, soybean oil, pumpkin seeds and pumpkin seed oil, purslane, perilla oil, nuts and nut oil [23].

However, most of these products are not included in the diet of an average consumer, the proportion of omega-3 fats is only $50-70 \%$ of the established norm. 
Thus, according to the survey conducted by the Institute of Nutrition of RAMS, the average consumption of fish and fish products in Russia is $9 \mathrm{~kg}$ per year per family member. This is lower than the recommended preventative level (30-40 g oily fish per day providing 600-800 mg omega -3 PUFA), in addition, not all fish products used contain sufficient quantity of omega -3 fatty acids [1].

Optimal daily consumption of omega-3 - 1 gram per day, omega-6 - 10 g, omega-7 and omega-9 - $30 \mathrm{~g}$. This amount is necessary for the normal functioning of the body cells.

As mentioned above, one of the sources of ingestion of unsaturated fatty acids is vegetable oils. Vegetable oils are used in food as an ingredient of various dishes, for example, as a dressing for salads, and are also the basis for the production of various types of oil and fat products.

Table 1 presents the content of unsaturated omega fatty acids in various types of food oils [23].

Table 1. Content of omega-3, omega-6 and omega-9 in oils.

\begin{tabular}{|l|c|c|c|c|}
\hline \multirow{2}{*}{ Type of oil } & \multicolumn{4}{c|}{ Amount of fatty acids, g per 100 g oil } \\
\cline { 2 - 5 } & omega -6 & omega -3 & omega -7 & omega -9 \\
\hline Olive oil & 9.76 & 0.76 & 1.26 & 71.58 \\
\hline Linseed oil & 12.70 & 52.50 & 0 & 21.0 \\
\hline Rape oil & 14.50 & 9.14 & 0.22 & 63.06 \\
\hline Mustard oil & 15.33 & 5.90 & 0.22 & 58.97 \\
\hline Peanut butter & 31.71 & 0 & 0.10 & 46.1 \\
\hline Sesame oil & 41.30 & 0.30 & 0.20 & 39.5 \\
\hline Walnut oil & 52.89 & 10.40 & 0.10 & 22.60 \\
\hline Corn oil & 53.52 & 1.16 & 0.11 & 27.46 \\
\hline Wheat germ oil & 54.80 & 6.90 & 0.50 & 14.60 \\
\hline Sunflower oil & 65.70 & 0 & 0 & 19.50 \\
\hline Grape seed oil & 69.59 & 0.10 & 1.20 & - \\
\hline Hempy oil & 40.00 & 15.00 & 0.15 & - \\
\hline Coconut oil & 1.68 & 0.02 & 0.02 & 6.32 \\
\hline
\end{tabular}

Analysis of the fatty acid composition of the presented oils shows that not all oils contain omega fatty acid of all groups. Thus, sunflower oil widely used in the diet does not contain omega-3 and omega-7 fatty acids in its composition. Being a leader in omega-3 fatty acids content, linseed oil does not contain omega-7 fatty acids. However, not absolute consumption, but the ratio between omega-6- and omega-3-fatty acids can have the crucial value.

For a healthy person it should be 10:1, in therapeutic nutrition 3:1 [21]. The oils that have the optimal or close to the optimal ratio of omega- 3 and omega- 6 fatty acids include linseed (4:1), mustard (1:2.6) and olive (1:13) oils.

Thus, no type of oil is able to fully fill the body's needs for unsaturated fatty acids. There is a need to provide the population with full-fledged, high-quality fat products taking into account their biological properties and metabolism in the body. Particular attention should be paid to products in the form of aqueous fat emulsions, as they are easily absorbed and contribute to the stabilization of physiological functions of the gastro-intestinal tract. 


\section{Research methodology}

Within the framework of the task on the basis of the vegetable food oils application, the basic design for production of a number of emulsion products characterized by an optimal ratio of omega- 3 and omega- 6 fatty acids and recommended consumption standards for mono- and polyunsaturated fatty acids was made.

At the development stage of the functional basis for food emulsions on the basis of the oils complex, a heuristic model was developed, specifying the initial and resultant design parameters (Fig. 2.).

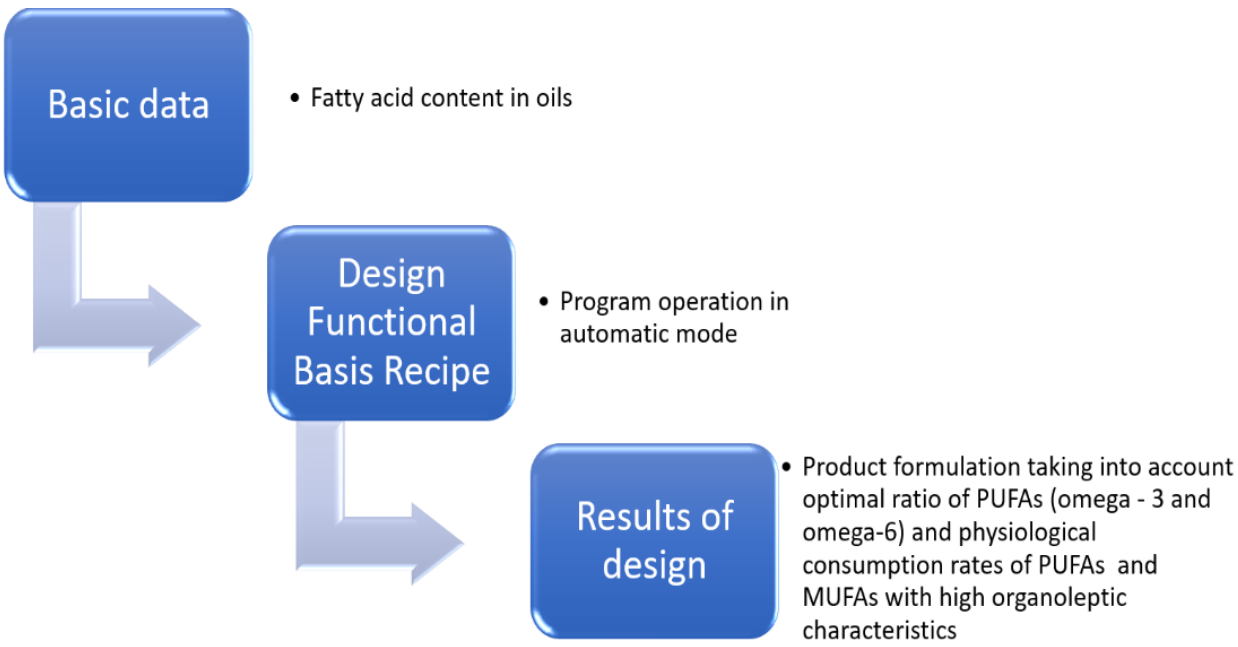

Fig. 2. Design model of functional basis for food emulsions

In the first step, this model involves the rapid access to an information database containing information on the fatty acid composition of various types of edible oils, the possibility of adjustment, adding and deleting data. The information is entered into the corresponding program by an operator. In the second stage in automatic mode, taking into account the purpose and the established design framework, the design of the functional fat base formulation is performed in automatic mode. The third step is intended to produce a specific design result, namely a finished formulation of a functional fat base containing the required ratio of omega - 3 and omega - 6 fatty acids, as well as optimized for the daily requirement of mono- and polyunsaturated fatty acids based on estimated consumption per day with high organoleptic characteristics.

\section{Experimental results}

An additional result of this phase of work was the creation of the "Design of Fatty Acid Ratio" program.

The program is designed to compile optimal formulations of the functional fat base, and optimization means the combination of oils based on the ratio of omega- 3 and omega- 6 fatty acids per $100 \mathrm{~g}$ of source oil.

The program allows you to quickly compile optimized formulations of products from a combination of available raw materials. Any number of oil types and the number of properties (characteristics) can be specified.

The program includes a database of omega-3 and omega- 6 fatty acids in various oils, which can be expanded both to the number of oils used and to the indicators, by which it is being optimized. 
Directly the software module contains: target function, raw material selection register, variable parameters, as well as a block of obtained results verification.

The objective function is the ratio of omega- 3 and omega- 6 amino acids in the optimized formulation:

$$
F(x) \rightarrow \sum \omega 3 / \sum \omega 6
$$

The result of the target function is specified by an operator as any valid value. Other parameters (including complex or integral indicators) may be added as an extension of the program functionality.

The raw material selection register determines the set of oils involved in the preparation of an optimized formulation from the base. Based on the register, the matrix of the main calculation and validation of results is formed.

The norm of oil laying in the optimized formulation ( $g$ ) is serving as changing parameters, which determines the actual content of fatty acids by dependence:

$$
X_{\Phi}=\frac{M_{n} \cdot X}{100}
$$

where $\mathrm{Mn}$ is the variable parameter of the norm of oil laying into the optimized formulation, $\mathrm{g}$;

$\mathrm{X}$ is the mass fraction of omega- 3 and omega- 6 fatty acids.

The verification unit contains a mathematical model of formulation calculation with the mass fraction (in \%) of each component, as well as the actual ratio of fatty acids per $100 \mathrm{~g}$.

The program is based on Microsoft Excel using the integrated language Visual Basic for Application. The solution of the problem is carried out by the method of generalized lowering gradient with the following parameters: restriction accuracy - 0.000001 , convergence -0.0001 . As an alternative, it is possible to solve the problem by simplex method.

The algorithm of work includes:

- selection of oils involved in solving the optimization problem (minimum value is 1 , maximum - number of records in the database);

- specifying the original norm of laying (default is 100);

- specifying the results of the target function.

Based on the entered data an information matrix is formed, which will participate in the solution of the problem. The matrix includes linear equations of calculating the formulation and mass fraction of each component (at the data entry stage, all components will be divided in equal proportions). After solving the problem, all calculations are repeated taking into account the specified target function.

As a solution to the problem, the following options are possible: the only solution is found, the solution is missing, there are several solutions. The only solution can be a laying value of one (or several oils) $0 \mathrm{~g}$, which is acceptable in the mathematical model, but not in the technological approach. Elimination of such situation is possible by specifying additional parameters like: Mn0, which automatically translates the solution to the "solution missing."

An example of solving the optimization problem is presented in Figure 3. 


\begin{tabular}{|c|c|c|c|c|c|c|c|c|c|c|}
\hline Type of oil & $\begin{array}{l}\text { Amount of fatty } \\
\text { acids omega }-6 \text {, } \\
\text { g per } 100 \mathrm{~g} \text { oil }\end{array}$ & \begin{tabular}{|} 
Amount of fatty \\
acids omega -3, \\
g per 100 g oil
\end{tabular} & $\begin{array}{l}\text { choice of } \\
\text { a product }\end{array}$ & weight & fact-6 & fact-3 & $\begin{array}{l}\text { forecast } \\
\text { of norm }\end{array}$ & $\begin{array}{c}\text { after } \\
\text { optimizati } \\
\text { on- } 6\end{array}$ & $\begin{array}{c}\text { after } \\
\text { optimizati } \\
\text { on-3 }\end{array}$ & $\begin{array}{c}\text { specific } \\
\text { weight, } \\
\%\end{array}$ \\
\hline Olive oil & 9,76 & 0,76 & & & & & 0 & & & 0 \\
\hline Linseed oil & 12,7 & 52,5 & & & & & 0 & & & 0 \\
\hline Rape oil & 14,5 & 9,14 & 1 & 100 & 14,5 & 9,14 & 1,959469 & 0,284123 & 0,179096 & 19,7 \\
\hline Mustard oil & 15,33 & 5,9 & 1 & 100 & 15,33 & 5,9 & 8 & 1,2264 & \begin{tabular}{|l|}
0,472 \\
\end{tabular} & 80,3 \\
\hline Peanut butter & 31,71 & 0 & & & & & 0 & & & 0 \\
\hline Sesame oil & 41,3 & 0,3 & & & & & 0 & & & 0 \\
\hline Walnut oil & 52,89 & 10,4 & & & & & 0 & & & 0 \\
\hline Corn oil & 53,52 & 1,16 & & & & & 0 & & & 0 \\
\hline Wheat germ oil & 54,8 & 6,9 & & & & & 0 & & & 0 \\
\hline Sunflower oil & 65,7 & 0 & & & & & 0 & & & 0 \\
\hline Grape seed oil & 69,59 & 0,1 & & & & & 0 & & & 0 \\
\hline Hempy oil & 40 & 15 & & & & & 0 & & & 0 \\
\hline Coconut oil & 1,68 & 0,02 & & & & & 0 & & & 0 \\
\hline
\end{tabular}

Fig. 3. Data matrix and resulting ratio of omega- 3 and omega- 6 fatty acids

At the user level, the dialogue with the program is reduced to entering commands getting the solution (formulation directly). The program can be used by catering and food industry enterprises to compile optimal recipe mixtures of oils for the purpose of producing various types of emulsion products, in particular, sauces.

Figure 3 shows one of the obtained solutions of the problem.

Two types of oil - rapeseed and mustard - were selected from the list of intended ingredients included in the information matrix by the operator. The default laying norm is set to 100 .

First, the program outputs the actual content of omega- 3 and omega- 6 fatty acids per $100 \mathrm{~g}$ of the product and the forecast of the norm. Then, on the basis of the information matrix and the solution of the system of linear balance equations taking into account constraints, as well as the target function to optimize the ratio of fatty acids in automatic mode a positive solution defining one product formulation was obtained: $19.7 \mathrm{~g}$ of rapeseed and $80.3 \mathrm{~g}$ of mustard oil. The choice of formulation in this case is determined by optimization of the target function based on the ratio of omega- 3 and omega- 6 fatty acids in the product being designed.

Further research will expand the information matrix of the program with indicators to optimize the formulation of the functional fat base based on the daily need for mono- and polyunsaturated fatty acids and organoleptic characteristics of the finished product.

\section{The discussion of the results}

Several researchers have already proven the role of mono- and polyunsaturated fatty acids in the prevention of some of the most common diseases today - cardiovascular. The main scientifically substantiated and confirmed effects of omega fatty acids, influencing physiological functions, metabolic processes in the human body are approved by regulatory documents: level control of glucose, triacylglycerins, cholesterol, lipoproteins of high and low density in blood, preservation of the tone of blood vessels walls and their passability, provision of systemic immuno-modelling action, antithrombotic action. In addition, there are data on the functions of unsaturated fatty acids such as protecting cells from external damage and strengthening their natural barrier, synthesizing critical substances exerting anti-inflammatory and anti-allergic action, etc. 
However, there is evidence that compliance with the standards of their use is important in achieving functional effects for the body derived from the consumption of omega fatty acids, and also recommended ratio of omega- 6 to omega-3 PUFA. In order to control the amount of unsaturated fatty acids consumed with food, it is necessary to monitor the diet. Creating food products balanced in the composition of omega fatty acids will solve this problem.

One of the sources of omega groups fatty acids supply to the body is with vegetable oils. Analysis of the chemical composition showed that not every oil contains the full omega group of fatty acid, and most of the oils do not meet the recommended ratio of omega- 6 to omega -3 PUFA.

To create a functional basis of emulsion products characterized by high degree of fats digestibility and stabilization of physiological functions of the human gastrointestinal tract, it is advisable to use a combination of different food vegetable oils with an optimum ratio of omega-6 to omega-3 PUFA.

To solve this problem, a model of designing a functional basis for food emulsions has been developed, including three stages, each of which is aimed at the implementation of the corresponding task. The result of the design is to obtain a finished product, the formulation of which is prepared taking into account the optimal ratio of PUFA (omega - 3 and omega6) and the physiological standards of consumption of PUFA and MUFA with high organoleptic characteristics.

In the framework of this model a design program of fatty acids ratio was developed, which is designed to compile optimal formulations of the functional fat base based on the ratio of omega- 3 and omega- 6 fatty acids per $100 \mathrm{~g}$ of the original oil. This program will make the formulation of the fat base, which has functional properties for the production of emulsion food products both for food service establishments and for food industry enterprises. Such products can be ready food products - emulsion sauces, including mayonnaise, spreads, etc., as well as raw materials used in the production of other food products.

On the basis of the researches it is planned to expand the parameters of formulation optimization taking into account the daily physiological need of the human body in functional food ingredients - mono- and polyunsaturated fatty acids, and considering the high organoleptic advantages of the finished product.

\section{References}

1. State Report on the State of Sanitary and Epidemiological Well-Being of the Population in the Russian Federation in 2019 , (2019)

2. A.V. Pogozheva, A.K. Baturin, E.U. Sorokina, T.N. Solntseva, M.M. Korosteleva, Food questions, 83, 32 (2014)

3. Christopher J Gerry, Maria Kaneva, Applied Research in Quality of Life (2020) https://doi.org/10.1007/s11482-020-09831-4

4. GOST R 52349-2005 Food products. Functional foodstuff. Terms and definitions. Standartinform, 1 (2006)

5. Standards of physiological requirements for energy and food substances for different population groups of the Russian Federation. Methodological recommendations: Moscow: Federal Center of Hygiene and Epidemiology of Rospotrebnadzor, 36 (2009)

6. GOST R 54059-2010 Functional food products. Ingredients food functional. Classification and general requirements Standartinform, 7 (2011) 
7. G.A. Dhopeshwarkar, J.F. Mead, J. of the American Oil Chemists Society, 38, 297 (1961)

8. S. Terés, G. Barceló-Coblijn, M. Benet, R. Alvarez, R. Bressani, ProcNatl Acad Sci USA, 105, 13811 (2008)

9. C. Carrillo, M. CaviaMdel, S. Alonso-Torre, Nutr Hosp, 27, 978 (2012)

10. Yang, Z. H., Miyahara, H., \& Hatanaka, A. Lipids in health and disease, 10(1), 120 (2011) doi:10.1186/1476-511X-10-120

11. I. Çimen, B. Kocatürk, S. Koyuncu, Ö. Tufanlı, U. I. Onat, A. D Yıldırım, Science Translational Medicine, 8 (358) (2016) doi:10.1126/scitranslmed.aaf9087

12. M. E. Frigolet, R. Gutiérrez-Aguilar, Advances in Nutrition: An Int. Rev. J., 8 (1), 173 (2017)

13. FDA announces qualified health claims for omega-3 fatty acids, Press release, (2004)

14. OBESITY Update June 2014, http://www.oecd.org/els/healthsystems/Obesity-Update2014.

15. Omega-3 supplements may slow a biological effect of aging, URL: https://www.sciencedaily.com/releases/2012/10/121001140957.htm

16. Research and Innovation Communications, http://researchnews.osu.edu/archive/omega3aging.htm

17. J.S. Volek, Lipid Research (2008)

18. D.S. Siscovick, Circulation, 114, 209 (2006)

19. J.S. Volek, Progress in Lipid Research, Restricting carbohydrates (2008)

20. L. Hooper, L. Al-Khudairy, A.S. Abdelhamid, Cochrane Database Syst. Rev. 11, CD011094 (2018)

21. A Simopoulos, Food Rev Int, 20, 77 (2004)

22. Fats and fatty acids in human nutrition. Expert Consultation Report, FAO Food and Nutrition Paper 91, FAO, Rome, (2010)

23. USDA. Food Data Central, https://fdc.nal.usda.gov/fdc-app.html\#/?query=oil 